\title{
La ozonoterapia en pacientes con enfisema pulmonar
}

\author{
JOSÉ LUIS CALUNGA F. ${ }^{1, a}$, YULEIDYS PAZ A. ${ }^{2}$, SILVIA MENÉNDEZ C. .,b,a, \\ ALFREDO MARTÍNEZ ${ }^{3}$, APARICIO HERNÁNDEZ ${ }^{3}$
}

\section{Rectal ozone therapy for patients with pulmonary emphysema}

Background: Ozone therapy may stimulate antioxidant systems and protect against free radicals. It has not been used formerly in patients with pulmonary emphysema. Aim: To assess the effects of rectal ozone therapy in patients with pulmonary emphysema. Material and Methods: Sixty four patients with pulmonary emphysema, aged between 40 and 69 years, were randomly assigned to receive rectal ozone in 20 daily sessions, rectal medicinal oxygen or no treatment. Treatments were repeated three months later in the first two groups. At baseline and at the end of the study, spirometry and a clinical assessment were performed. Results: Fifty patients completed the protocol, 20 receiving ozone therapy, 20 receiving rectal oxygen and 10 not receiving any therapy. At baseline, patients on ozone therapy had significantly lower values of forced expiratory volume in the first second $\left(F E V_{1}\right)$ and FEV $/$ Forced vital capacity. At the end of the treatment period, these parameters were similar in the three treatment groups, therefore they only improved significantly in the group on ozone therapy. No differences were observed in other spirometric parameters. Conclusions: Rectal ozone therapy may be useful in patients with pulmonary emphysema.

(Rev Med Chile 2011; 139: 439-447).

Key words: Bronchospirometry; Emphysema; Ozone.

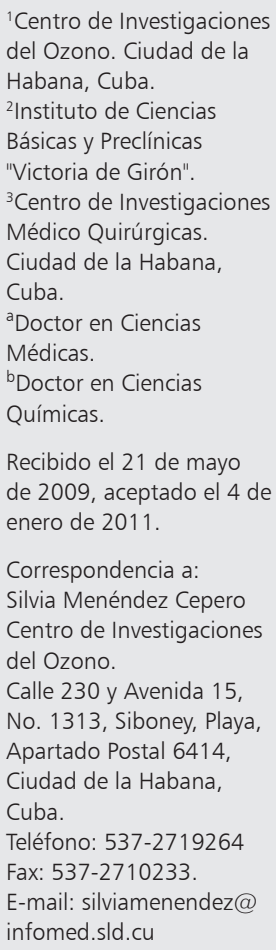

L a enfermedad pulmonar obstructiva crónica (EPOC) se caracteriza por limitación progresiva al flujo del aire, no totalmente reversible, asociada con una respuesta inflamatoria anormal a partículas o gases nocivos. Clásicamente se identifican dos fenotipos: pacientes con EPOC en los que predomina la destrucción -enfisema-o bien, el caso de pacientes que predomina la obstrucción bronquial -bronquitis crónica-.

El enfisema se define anatómicamente como una distensión permanente y destructiva de los espacios aéreos distales a los bronquiolos terminales, con fibrosis evidente y pérdida de la arquitectura normal ${ }^{1-3}$. En la práctica clínica, un paciente con enfisema ha sido o es un fumador importante, no obstante, en determinados países existen factores ambientales, que pueden ser la principal causa de desarrollo de la enfermedad ${ }^{4-6}$.
La triada de síntomas principales del enfisema son: disnea, tos y expectoración, siendo la disnea el síntoma que más incomoda y angustia al paciente. Aunque al enfisema se define en términos patológicos es importante también el empleo de técnicas como las de radiografía y la tomografía axial computarizada (TAC) de tórax, ya que estas ponen en evidencia las alteraciones morfológicas atribuibles a esta enfermedad ${ }^{7-9}$.

El tratamiento de estos pacientes debe ser multidisciplinario e individualizado teniendo como objetivos básicos frenar la progresión de la enfermedad, aliviar los síntomas y prevenir o corregir las complicaciones o reagudizaciones que aparezcan. Tradicionalmente, el tratamiento se basa en el empleo de broncodilatadores y aún hoy siguen siendo el tratamiento básico de la enfermedad. No obstante, las nuevas concepciones etiopatogénicas 
(base inflamatoria), suponen un enfoque terapéutico diferente, introduciéndose más recientemente el empleo de antiinflamatorios ${ }^{10-12}$.

El ozono es un gas extremadamente reactivo y su mecanismo de acción está relacionado con la generación de productos secundarios que en cantidades adecuadas ejercen diversas acciones biológicas que le confieren sus propiedades terapéuticas, tales como: germicida, modulador del estrés oxidativo y del sistema inmunológico y mejora el flujo sanguíneo. Además, el ozono incrementa la presión parcial de oxígeno en sangre, en tejido, sobre el metabolismo aeróbico mejora la respiración celular ya que actúa estimulando la actividad de enzimas como la de glucosa-6-fosfato-deshidrogenasa. A nivel preclínico se ha observado una modulación a nivel de tejido pulmonar lesionado de la enzima mieloperoxidasa. Todos estos aspectos hacen pensar que la aplicación del ozono en pacientes con EPOC, podría tener un efecto beneficioso ${ }^{13-21}$.

Se reconoce que un estrés oxidativo moderado y controlado induce defensa enzimática antioxidante por lo que se ha señalado al precondicionamiento oxidativo como un mecanismo que pudiera explicar las acciones farmacológicas del ozono en las enfermedades mediadas por las especies reactivas del oxígeno $(E R O)^{1316-21}$. En el trastorno patológico del enfisema se destaca el papel del estrés oxidativo y su implicación en la aparición de la inflamación ${ }^{2,11,12,22}$.

En el momento actual, existen millones de personas diagnosticados de enfisema pulmonar y muchos de estos individuos sufren de discapacidad progresiva. Además, a esto se le suma que el número de muertes por año atribuidas a esta enfermedad sigue aumentando. Por tanto, existe una creciente necesidad de mejorar la calidad de vida y el manejo terapéutico del paciente con enfisema pulmonar. Es por ello que el objetivo de nuestro trabajo es estudiar los efectos de la ozonoterapia sobre los síntomas y el estatus de salud de pacientes con enfisema pulmonar.

\section{Materiales y Métodos}

Se realizó un ensayo clínico aleatorizado, doble ciego, en 50 pacientes con diagnóstico de enfisema pulmonar. Se incluyeron pacientes ambulatorios, de ambos sexos, entre 40 y 69 años, con consentimiento informado firmado en una planilla titulada
Acta de consentimiento para el estudio. Además, los pacientes tenían que haber dejado el hábito tabáquico, 5 años antes como mínimo.

Se excluyeron pacientes con: enfermedades crónicas descompensadas o malignas con actividad, embarazo y puerperio, cirugía mayor, traumatismo recientes y pacientes psiquiátricos. Fueron excluidos pacientes asmáticos a los cuales se les realizo una espirometría con y sin el uso de broncodilatador. Entre los criterios de salida se consideraron la salida voluntaria del estudio, el no cumplimiento del tratamiento, la aparición de enfermedad aguda de etiología infecciosa u de otra causa, la aparición de reacciones adversas que modificaran el tratamiento o el fallecimiento.

Este estudio fue aprobado por el Consejo Científico y el Comité de Ética del Centro de Investigaciones del Ozono.

Los pacientes con enfisema pulmonar fueron divididos en tres grupos de estudio-tratamiento:

Grupo 1: Constituido por 20 pacientes con tratamiento de base más administración de ozono por vía rectal, a una dosis de $6 \mathrm{mg}(30 \mathrm{mg} / \mathrm{L}$ de concentración y $200 \mathrm{~mL}$ de volumen)/día, 20 sesiones, seguido de 3 meses de descanso y luego un segundo ciclo de 20 sesiones más.

Grupo 2: Igual al grupo 1, pero aplicando oxígeno medicinal en vez de ozono por igual vía.

Grupo 3: Se tomó como grupo control positivo a 10 pacientes que sólo mantuvieron su tratamiento de base y evolucionó el mismo tiempo del resto de los grupos.

Los diferentes esquemas del tratamiento basal fueron ${ }^{1,4,9}$ :

1. Salbutamol, bromuro de ipratropio y prednisona.

2. Salbutamol, aminofilina y prednisona.

3. Salbutamol, beclometasona, bromuro de ipratropio y prednisona.

4. Salbutamol, bromuro de ipratropio y aminofilina.

\section{Proceder para la aplicación de la ozonoterapia}

El ozono se obtuvo de un equipo generador de ozono de uso medicinal llamado Ozomed 401, el gas ozono fue generado a partir del oxígeno medicinal acoplado a este equipo generador donde se extrajo una mezcla ozono/oxígeno la cual fue aplicada por vía rectal empleando sonda rectal Nelaton calibre 10, previo cálculo de la dosis mediante la concentración y el volumen de la mezcla. 


\section{Fundamentos del uso de la vía rectal para la ozonoterapia}

Diferentes estudios, tanto preclínicos como clínicos han demostrado el papel de la ozonoterapia por vía rectal en la estimulación del metabolismo del oxígeno, ya mencionado. Así también, en la activación de la actividad antioxidante endógena, como inmunoregulador, y su efecto en la elevación en sangre del 2,3 disfosfoglicerato, por esta vía. Por el contrario, la ozonoterapia no es recomendable por vía inhalatoria ya que en altas concentraciones es tóxica, sobre todo para el sistema respiratorio ${ }^{13-19}$

En cada uno de los grupos hubo una distribución homogénea de los pacientes según el tratamiento de base que estos llevaban. La duración del estudio se extendió por cinco meses. $\mathrm{Ni}$ el médico ni los pacientes conocían a que grupo de tratamiento pertenecían. La enfermera fue la encargada de asignar a los pacientes en forma aleatorizada -mediante una lista- a uno de los 3 grupos de tratamiento en forma consecutiva.

Con el objetivo de valorar la eficacia del método terapéutico se realizó una evaluación al inicio y al finalizar el segundo ciclo de tratamiento con ozono y oxígeno, así como en el grupo control, que consistió en la realización de:

\section{Pruebas funcionales respiratorias}

Se evaluaron a través de un espirómetro marca CHESTAC 25 acoplado a una microcomputadora (programa INTESP-2). Los parámetros evaluados fueron:

- $\mathrm{VEF}_{1}$ (Volumen espiratorio forzado en el primer segundo): Volumen de aire eliminado en el primer segundo de una capacidad forzada. Se considera la existencia de alteraciones obstructivas cuando el valor obtenido es inferior al $75 \%$ del valor predicho (valores obtenidos en una población sana, comparable según edad, peso, sexo y talla, tomado estos datos de pruebas funcionales de pacientes aparentemente sanos de una base de datos del Centro de Investigaciones Médico-Quirúrgicas de Ciudad de La Habana).

- CVF (Capacidad vital forzada): Volumen gaseoso máximo que puede ser expedido de los pulmones durante un esfuerzo máximo, precedido de una inspiración forzada efectuada de una forma rápida en 3 ó 4 segundos.
- $\mathrm{FEF}_{25-75 \%}$ (Flujo medio máximo espiratorio): Cantidad de aire que se espira, existente en la parte media de la curva espirográfica de la capacidad vital forzada, resulta independiente del esfuerzo del individuo y expresa la salida del aire contenido en las ramificaciones finas del árbol bronquial (si está alterado refleja obstrucción de las vías aéreas de mediano y pequeño tamaño).

- $\mathrm{VEF}_{1} / \mathrm{CVF}$ (Índice de Tiffeneau): Relación entre la capacidad vital forzada que se espira en el primer segundo y la capacidad vital forzada, es de aproximadamente el $80 \%$ (si está disminuido demuestra la existencia de un proceso obstructivo).

Para la realización de estas pruebas se tuvo en cuenta que el paciente estuviera clínicamente estable y no ingiriese sus medicamentos de base, $24 \mathrm{~h}$ antes de realizadas las pruebas espirométricas, que fuera durante la mañana y con una alimentación ligera. Además, se midió el nivel de hemoglobina y el pH sanguíneo y la gasometría arterial (presión parcial de anhídrido carbónico- $\mathrm{PaCO}_{2}$ y presión parcial de oxígeno- $\mathrm{PaO}_{2}$ ) y las pruebas imagenológicas (radiografía y TAC de tórax).

Se determinó la respuesta clínica evolutiva de los pacientes, de acuerdo a su valoración subjetiva, según espaciamiento de los síntomas más frecuentes: disnea, tos y sibilancias ${ }^{23,24}$. Se clasificó a los pacientes después del tratamiento en mejorado, cuando existía una disminución de los síntomas; estable, cuando se mantenían y empeorado, cuando aumentaban.

También se tuvo en cuenta la valoración clínica según la espirometría. Con la prueba inicial se clasificaron a los pacientes de acuerdo al grado de obstrucción (clasificación de la American Thoracic Society) en $\left({ }^{1,4,4,25}\right)$ :

- Ligero: $\mathrm{FEV}_{1}$ entre $85-70 \%$ del valor de referencia.

- Moderado: Entre 60-69\%.

- Moderadamente severo: Entre 50-59\%.

- Severo: entre 34-49\%.

- Muy severo: Inferior al 34\%.

Al final del estudio se repitió la espirometría y se evaluaron los pacientes, teniendo en cuenta que para pasar de un grado de obstrucción a otro anterior, era necesario un aumento del FEV igual o superior a $12 \%$ del nivel basal (valor inicial). De 
acuerdo a esto se clasificó en: mejorado, paciente que pasa del grado de obstrucción con que se clasificó a un grado menor; estable, paciente que continúa en el mismo grado de obstrucción y empeorado, paciente que pase del grado de obstrucción con que se clasificó a un grado superior.

Se estudio la evolución de parámetros índice de BODE el cual es una medida íntegra para la evolución del paciente con $\mathrm{EPOC}^{26}$, este índice mide: 1) El $\mathrm{FEV}_{1}$; 2) la distancia caminada en 6 minutos, la cual debe ser mayor de 350 metros; 3) el grado de disnea y 4) el índice de masa corporal expresado en $\mathrm{kg} / \mathrm{m}^{2}$ de superficie corporal (Tabla 8 ). Este índice describe 4 grados de severidad que va desde valor 0 el cual representa baja mortalidad hasta el valor 3 que predice alta mortalidad para estas 4 variables:

\section{Estadística}

Se utilizó en variables cuantitativas la prueba $t$ de Student para comparar dos medias aritméticas y para comparar variables cualitativas se utilizó la prueba $\chi^{2}$, ambas con un nivel de significación de $5 \%$.

\section{Resultados}

Inicialmente fueron incluidos en el estudio un total de 64 pacientes de los cuales 14 cumplían criterios de salida, los que incluían, abandono voluntario del estudio y no cumplimiento adecuado del tratamiento.

Los 50 pacientes restantes fueron evaluados y tenían una edad entre 40 y 69 años. La distribución de los tratamientos de base según los grupos de estudio fue homogénea (Tabla 1). Respecto al resultado de las pruebas funcionales respiratorias, se alcanzaron aumentos significativos, entre el valor inicial y final, en los valores de la $\mathrm{FEV}_{1}$ y $\mathrm{FEV}_{1} /$ CVF en el grupo Ozono, no así en los otros grupos. En cuanto a los valores de la CVF y el $\mathrm{FEF}_{25-75 \%}$ no hubo modificación en ninguno de los grupos, sólo se obtuvieron discretos aumentos en el grupo Ozono, sin llegar a ser estadísticamente significativos (Tabla 2). La hemoglobina experimentó una disminución significativa en el grupo Ozono, sin embargo, mantuvo estable sus valores en los otros grupos (Tabla 3 ).

En la Tabla 4 se reflejan el comportamiento de los parámetros gasométricos y el $\mathrm{pH}$. Se observa un aumento significativo sólo en la $\mathrm{PO}_{2}$ en el grupo 1 (Ozono). $\mathrm{La}^{\mathrm{PCO}_{2}}$ y el $\mathrm{pH}$ mantuvieron sus valores sin diferencias significativas en los diferentes grupos de tratamiento.

En cuanto a los estudios imagenológicos, la TAC de tórax reflejó presencia de enfisema pulmonar difuso en el total de la muestra, y se mantuvo sin cambio al final del tratamiento. También se observaron otros signos tales como: tractos fibrosos asociados, áreas de hipoaereación y fibro enfisema, que aunque no estaban presentes en todos los pacientes, se mantuvieron al final del tratamiento, en todos los grupos. Respecto a la radiografía de tórax, en $100 \%$ de la muestra aparecieron signos de enfisema, sin modificaciones en ninguno de los grupos, al final del estudio. También se observaron reforzamiento en la trama del hilio, radiotransparencia en ambas bases pulmonares y aumento del diámetro ántero-posterior en algunos de los pacientes, sin cambios al final del tratamiento, en ninguno de los grupos.

Tabla 1. Distribución de los tratamientos de base según grupo de estudio

\begin{tabular}{|c|c|c|c|c|c|c|c|c|}
\hline \multirow{2}{*}{$\begin{array}{l}\text { Tratamientos } \\
\text { de base }\end{array}$} & \multicolumn{2}{|c|}{ Ozono } & \multicolumn{2}{|c|}{ Oxígeno } & \multicolumn{2}{|c|}{ Control } & \multicolumn{2}{|c|}{ Total } \\
\hline & & (\%) & & (\%) & & (\%) & $\mathbf{n}$ & (\%) \\
\hline 1 & 6 & (30) & 6 & (30) & 3 & (30) & 15 & (30) \\
\hline 2 & 5 & (25) & 5 & (25) & 2 & (20) & 12 & (24) \\
\hline 3 & 5 & (25) & 5 & (25) & 3 & (30) & 13 & (26) \\
\hline 4 & 4 & (20) & 4 & (20) & 2 & (20) & 8 & (16) \\
\hline Total & 20 & (100) & 20 & (100) & 10 & (100) & 50 & (100) \\
\hline
\end{tabular}

Esquema 1 de tratamiento de base estaba conformado por salbutamol, bromuro de ipratropio y prednisona; el esquema 2 por salbutamol, aminofilina y prednisona; el esquema 3 por beclometasona, bromuro de ipratropio, aminofilina y prednisona y el esquema 4 por salbutamol, bromuro de ipratropio y aminofilina. La distribución de los tratamientos de base por grupo fue homogénea, $p>0,05$. 
Tabla 2. Comportamiento de las pruebas funcionales respiratorias, al inicio y al final del tratamiento, para los diferentes grupos de estudio

\begin{tabular}{|c|c|c|c|c|c|c|}
\hline \multirow[t]{2}{*}{ Espirometría } & \multicolumn{2}{|c|}{ Ozono } & \multicolumn{2}{|c|}{ Oxígeno } & \multicolumn{2}{|c|}{ Control } \\
\hline & Inicio & Final & Inicio & Final & Inicio & Final \\
\hline $\operatorname{VEF}_{1}(\%)$ & $51,2 \pm 6,0$ & $59,3 \pm 6,3$ & $58,2 \pm 4,3$ & $59,1 \pm 4,8$ & $59,2 \pm 4,1$ & $59,8 \pm 4,5$ \\
\hline Prueba t & $t=15,3$ & $p<0,0005$ & NS & NS & NS & NS \\
\hline $\mathrm{VEF}_{1} / \mathrm{CVF}(\%)$ & $54,4 \pm 4,1$ & $63,4 \pm 4,2$ & $60,2 \pm 3,4$ & $61,6 \pm 3,1$ & $61,2 \pm 2,8$ & $61,3 \pm 2,5$ \\
\hline Prueba $t$ & $t=17,4$ & $p<0,0005$ & NS & NS & NS & NS \\
\hline CVF (\%) & $94,0 \pm 2,1$ & $93,7 \pm 2,9$ & $95,6 \pm 5,3$ & $95,4 \pm 4,8$ & $96,5 \pm 3,4$ & $95,6 \pm 3,6$ \\
\hline Prueba t & NS & NS & NS & NS & NS & NS \\
\hline $\mathrm{FEF}_{25-75 \%}(\mathrm{~L} / \mathrm{s})$ & $1,35 \pm 0,11$ & $1,42 \pm 0,14$ & $1,39 \pm 0,15$ & $1,40 \pm 0,16$ & $1,41 \pm 0,13$ & $1,42 \pm 0,14$ \\
\hline Prueba $t$ & NS & NS & NS & NS & NS & NS \\
\hline
\end{tabular}

VEF : Volumen espiratorio forzado en el primer segundo; VEF $/$ CVF (Índice de Tiffenau): Porcentaje de la CVF que se espira en el primer segundo partido por la CVF; CVF -Capacidad vital forzada; $\mathrm{FEF}_{25-75 \%}$ : Flujo medio máximo espiratorio; NS: no significativo. .

En la Tabla 5 se observan las valoraciones subjetivas de los pacientes al final del tratamiento. Se aprecia que el grupo Ozono tuvo el mayor número de pacientes mejorados (13), seguido del grupo Oxígeno (6), sin diferencias significativas entre ambos tratamientos; el grupo control tuvo el menor número de casos mejorados (1) con diferencias significativas respecto al grupo Ozono. Pacientes empeorados sólo se observaron en los grupos Oxígeno y Control, con un caso cada uno.
Tabla 3. Valores de hemoglobina, al inicio y al final del tratamiento, para los diferentes grupos de estudio

\begin{tabular}{|lccc|}
\hline Grupos & $\begin{array}{c}\mathbf{H b}(\mathbf{g} / \mathbf{L}) \\
\text { Inicio }\end{array}$ & $\begin{array}{c}\mathbf{H b}(\mathbf{g} / \mathbf{L}) \\
\text { Final }\end{array}$ & $\mathbf{p}$ \\
Ozono & $\begin{array}{c}14,2 \pm 1,3 \\
\mathrm{t}=10,2\end{array}$ & $13,6 \pm 1,2$ & $<0,005$ \\
Oxígeno & $13,3 \pm 1,0$ & $13,0 \pm 1,1$ & NS \\
Control & $13,4 \pm 1,0$ & $13,3 \pm 1,0$ & NS \\
\hline
\end{tabular}

$\mathrm{Hb}$ : hemoglobina; NS: no significativo.

Tabla 4. Comportamiento de los parámetros gasométricos y del pH en los diferentes grupos de tratamiento

\begin{tabular}{|c|c|c|c|c|c|c|}
\hline & \multicolumn{2}{|c|}{ Ozono } & \multicolumn{2}{|c|}{ Oxígeno } & \multicolumn{2}{|c|}{ Control } \\
\hline & Inicio & Final & Inicio & Final & Inicio & Final \\
\hline $\mathrm{PaCO}_{2}(\mathrm{~mm} \mathrm{Hg})$ & $43,7 \pm 4,8$ & $43,3 \pm 3,6$ & $41,2 \pm 4,3$ & $41,0 \pm 3,2$ & $40,4 \pm 3,2$ & $40,3 \pm 3,0$ \\
\hline $\mathrm{p}$ & \multicolumn{2}{|c|}{ NS } & \multicolumn{2}{|c|}{ NS } & \multicolumn{2}{|c|}{ NS } \\
\hline $\mathrm{PaO}_{2}(\mathrm{~mm} \mathrm{Hg})$ & $64,8 \pm 6,6$ & $79,6 \pm 6,8$ & $71,1 \pm 7,2$ & $74,2 \pm 6,9$ & $72,3 \pm 6,5$ & $72,5 \pm 6,6$ \\
\hline $\mathrm{p}$ & \multicolumn{2}{|c|}{$p<0,005$} & \multicolumn{2}{|c|}{ NS } & \multicolumn{2}{|c|}{ NS } \\
\hline $\mathrm{pH}$ & $7,44 \pm 0,93$ & $7,43 \pm 0,91$ & $7,41 \pm 0,91$ & $7,41 \pm 0,91$ & $7,39 \pm 0,87$ & $7,40 \pm 0,90$ \\
\hline $\mathrm{p}$ & \multicolumn{2}{|c|}{ NS } & \multicolumn{2}{|c|}{ NS } & \multicolumn{2}{|c|}{ NS } \\
\hline
\end{tabular}

$\mathrm{PaCO}_{2}$ : Presión parcial de anhídrido carbónico en sangre arterial. $\mathrm{PaO}_{2}$ : Presión parcial de oxígeno en sangre arterial; NS: no significativo. 
En la evaluación clínica del paciente al final del estudio, según la espirometría, se observó que el grupo Ozono fue el único que presentó pacientes mejorados (11), con diferencias significativas con respecto al resto de los grupos. Sólo los grupos Oxígeno y Control reportaron pacientes empeorados, uno por grupo (Tabla 6).

Con respecto a la evolución de las variables analizadas en el índice de BODE (Tabla 7) observamos lo siguiente: 1) el grupo tratado con ozono incrementó el valor de $\mathrm{FEV}_{1}$ significativamente con respecto al grupo control y al grupo tratado con oxígeno al final del estudio;
2) en relación con la distancia caminada en 6 minutos, los pacientes tratados con ozono en este tiempo recorrieron mayor distancia de forma significativa comparado con los otros grupos, es de señalar que esta prueba fue realizadas por técnicos de rehabilitación que no conocían a que grupo correspondía cada paciente, 3) referente al grado de disnea el grupo que fue tratado con ozono disminuyó significativamente este variable al finalizar el estudio (Tabla 7); y 4) el índice de masa corporal, no sufrió variación ni al inicio, ni al final de nuestro estudio en los tres grupos, por eso no se refleja en la Tabla 7.

Tabla 5. Valoración subjetiva de los pacientes al final del tratamiento de acuerdo al grupo de estudio

\begin{tabular}{|lrrrrrrrr|}
\hline Evaluación clínica & \multicolumn{2}{c}{ Ozono } & \multicolumn{2}{c}{ Oxígeno } & \multicolumn{2}{c|}{ Control } & \multicolumn{2}{c|}{ Total } \\
& $\mathbf{n}$ & $\mathbf{( \% )}$ & $\mathbf{n}$ & $\mathbf{( \% )}$ & $\mathbf{n}$ & $\mathbf{( \% )}$ & $\mathbf{n}$ & $\mathbf{( \% )}$ \\
Mejorado & 13 & $(65)$ & 6 & $(30)$ & 1 & $(10)$ & 20 & $(40)$ \\
Estable & 7 & $(35)$ & 13 & $(65)$ & 8 & $(80)$ & 28 & $(56)$ \\
Empeorado & 0 & $(0)$ & 1 & $(5)$ & 1 & $(10)$ & 2 & $(4)$ \\
Total & 20 & $(100)$ & 20 & $(100)$ & 10 & $(100)$ & 50 & $(100)$ \\
\hline
\end{tabular}

Pacientes mejorados: $\chi^{2}=1,77 ; p=0,18$ (1 grado de libertad) Ozono vs Oxígeno. Pacientes mejorados: $\chi^{2}=8,01 ; p=0,0046$ (1 grado de libertad) Ozono vs Control.

Tabla 6. Evaluación clínica según espirometría al final del tratamiento de acuerdo al grupo de estudio

\begin{tabular}{|lrrrrrrrr|}
\hline Evaluación clínica & \multicolumn{2}{c}{ Ozono } & \multicolumn{2}{c}{ Oxígeno } & \multicolumn{2}{c|}{ Control } & \multicolumn{2}{c|}{ Total } \\
Mejorado & $\mathbf{n}$ & $\mathbf{( \% )}$ & $\mathbf{n}$ & $\mathbf{( \% )}$ & $\mathbf{n}$ & $\mathbf{( \% )}$ & $\mathbf{n}$ & (\%) \\
Estable & 11 & $(55)$ & 0 & $(0)$ & 0 & $(0)$ & 11 & $(22)$ \\
Empeorado & 9 & $(45)$ & 19 & $(95)$ & 9 & $(90)$ & 37 & $(74)$ \\
Total & 0 & $(0)$ & 1 & $(5)$ & 1 & $(10)$ & 2 & $(4)$ \\
\hline
\end{tabular}

Pacientes mejorados: $\chi^{2}=9,05 ; p=0,0026$ (1 grado de libertad) Ozono vs Oxígeno y Control.

Tabla 7. Evolución de variables medidas en el Índice de BODE al inicio y final del estudio

\begin{tabular}{|c|c|c|c|c|c|c|}
\hline Variable & $\begin{array}{l}\text { Inicio } \\
\text { Control }\end{array}$ & $\begin{array}{l}\text { Final } \\
\text { Control }\end{array}$ & $\begin{array}{l}\text { Inicio } \\
\text { Oxígeno }\end{array}$ & $\begin{array}{c}\text { Final } \\
\text { Oxígeno }\end{array}$ & $\begin{array}{l}\text { Inicio } \\
\text { Ozono }\end{array}$ & $\begin{array}{l}\text { Final } \\
\text { Ozono }\end{array}$ \\
\hline $\begin{array}{l}\mathrm{FEV} \\
\text { (\% valor predicho) }\end{array}$ & $59,2 \pm 4,1^{a}$ & $59,8 \pm 4,5^{a}$ & $58,2 \pm 4,3^{a}$ & $59,1 \pm 4,8^{a}$ & $51,2 \pm 6,0^{a}$ & $59,3 \pm 6,3^{b}$ \\
\hline $\begin{array}{l}\text { Distancia caminata } \\
6 \text { minutos }(\mathrm{m})\end{array}$ & $132,7 \pm 43,6^{a}$ & $141,5 \pm 57^{a}$ & $178,4 \pm 65^{a}$ & $182,4 \pm 66^{a}$ & $131,8 \pm 51^{a}$ & $174,8 \pm 62^{b}$ \\
\hline Disnea & $3,97 \pm 1,2^{a}$ & $3,7 \pm 1,3^{\mathrm{a}}$ & $3,2 \pm 1,3^{\mathrm{a}}$ & $2,8 \pm 0,7^{a}$ & $3,9 \pm 1,1^{\mathrm{a}}$ & $2,4 \pm 0,6^{b}$ \\
\hline
\end{tabular}

Letras a y b significan diferencias significativas entre dos evaluaciones; $p<0,05$. 
Ozonoterapia en enfisema pulmonar - J. L. Calunga et all

Tabla 8. Índice de BODE, que evalúa 4 variable en el paciente portador de EPOC

\begin{tabular}{|lrrrr|}
\hline Variable & $\mathbf{0}$ & $\mathbf{1}$ & $\mathbf{2}$ & $\mathbf{3}$ \\
FEV 1 (\% Valor predicho) & $\geq 65$ & $50-64$ & $36-49$ & $\leq 35$ \\
Distancia andada en 6 minutos $(\mathrm{m})$ & $\geq 350$ & $250-349$ & $150-249$ & $\leq 149$ \\
Disnea & $0-1$ & 2 & 3 & 4 \\
Índice de masa corporal $\mathrm{kg} / \mathrm{m}^{2}$ & $>21$ & $\leq 21$ & & \\
\hline
\end{tabular}

1) Función pulmonar $\left(\mathrm{FEV}_{1}\right)$; 2) Sintomatología (Disnea); 3) Capacidad de ejercicio (Prueba de caminata de 6 minutos) y 4) Estado nutricional (Índice de masa corporal).

\section{Discusión}

Los principales hallazgos encontrados en nuestro estudio son: que los pacientes con enfisema pulmonar y tratados con ozono presentaron un aumento significativo de los valores espirométricos, mejoria de la oxigenación y reducción de la sintomatología clínica.

$\mathrm{Al}$ analizar las pruebas funcionales respiratorias se observó que el grupo Ozono mostró un aumento significativo de $\mathrm{VEF}_{1}$ y $\mathrm{FEV}_{1} / \mathrm{CVF}$, lo cual está en correspondencia con estudios realizados en modelos experimentales en cobayos, donde en los grupos tratados con ozono se obtuvo una marcada disminución de la presión intrabronquial ${ }^{27,28}$.

También, en estudios de pacientes con asma moderada tratados con ozono por vía rectal y autohemoterapia mayor (durante 3 ciclos) se reflejaron aumentos significativos del $\mathrm{VEF}_{1}$ y CVF, junto con una disminución de los niveles sanguíneos de IgE y un aumento del sistema antioxidante del glutatión eritrocitario, corroborando que la ozonoterapia puede ser una alternativa de tratamiento en el asma bronquial, gracias a sus propiedades inmunomoduladoras y reguladoras del estrés oxidativo ${ }^{29}$.

Algunos autores plantean que en la EPOC, la presencia de reversibilidad en la obstrucción al flujo aéreo en ocasiones no se acompaña de cambios relevantes en la espirometría forzada, frecuentemente los pacientes refieren una clara mejoría clínica sin que se observe un incremento igual o superior al $12 \%$ en $\mathrm{VEF}_{1}$. De hecho, múltiples estudios han demostrado que puede haber cambios en la resistencia de la vía aérea, en la conductancia específica, en la capacidad residual funcional o en el volumen residual, sin una mejoría paralela en los parámetros espirométricos ${ }^{3,4,8,11}$. En nuestro estudio hubo mejoría, no de todos los parámetros de la función respiratoria, pero en cambio 13 pacientes tratados con ozono refirieron mejoría de su sintomatología. Se considera que la acción inmunológica del ozono a nivel sanguineo va dirigida, fundamentalmente, sobre los monocitos y linfocitos $\mathrm{T}$ que liberan pequeñas cantidades de diversas citocinas, particularmente del interferón gamma, pero también otras citocinas, como la $\mathrm{IL}_{10}$ y el factor transformador del crecimiento $\beta_{1}$ capaces de reducir la citotoxicidad autoreactiva. De esta forma las citocinas no sobrepasan los niveles necesarios, una vez que se activen de manera fisiológica los elementos contrareguladores ${ }^{14,21,30}$. De esta manera podemos ver el efecto inmunoregulador de la ozonoterapia, el cual contribuye a la disminución del proceso inflamatorio en nuestra patología ${ }^{31}$ y a la mejoría de la función pulmonar. Además, la ozonoterapia posee un poderoso efecto estimulador de las defensas antioxidantes ${ }^{13,16-21}$, que impide la progresión del proceso destructivo del tejido pulmonar.

Al hacer la valoración final de la hemoglobina, se observó una disminución significativa en el grupo Ozono, no así en los grupos oxígeno y control. Los pacientes con enfisema severo desarrollan una policitemia secundaria, como respuesta compensadora a la hipoxemia ${ }^{1,4,11}$. Esto concuerda con los resultados obtenidos en nuestro estudio, donde el grupo Ozono presentó al inicio cifras de $\mathrm{Hb}$ algo más elevadas, debido a que es el grupo con cifras de $\mathrm{PaO}_{2}$ más bajas (en la selección de los pacientes de este grupo coincidieron pacientes con mayor grado de obstrucción). Esto se debe al mecanismo compensatorio de la eritropoyetina estimulada por las bajas cifras de $\mathrm{PaO}_{2}$ que promueve la eritropoyesis. Al final del tratamiento, con la mejoría de la función pulmonar, este grupo presentó una dismi- 
nución significativa de las cifras de hemoglobina y un aumento significativo de la $\mathrm{PaO}_{2}$, comparado con el resto de los grupos.

La $\mathrm{PaCO}_{2}$ se encontró dentro de los límites normales, tanto al inicio como al final del estudio en los tres grupos. Lo mismo para el pH, coincidiendo con lo reportado en la literatura y con los mecanismos reguladores del equilibrio ácido/base con que cuenta nuestro organismo ${ }^{32}$.

El ozono es capaz de aumentar la flexibilidad del glóbulo rojo y la producción del 2,3 disfosfoglicerato, que actúa sobre la oxihemoglobina desplazando el equilibrio hacia la liberación de oxígeno a los tejidos ${ }^{13,21}$. Estos efectos se relacionan con los resultados obtenidos y muestran los beneficios de la ozonoterapia.

Respecto a los estudios imagenológicos, los signos de enfisema no mostraron modificaciones, corroborándose el componente irreversible del daño estructural en el enfisema pulmonar ${ }^{2,5,7}$.

Otros beneficios que se logran con la aplicación del ozono, es la reducción (en el 40\% de los casos) de la sintomatología clínica en estos pacientes, permitiéndoles referir una mejoría en cuanto a su autovaloración. Resultados similares se obtuvieron en pacientes asmáticos tratados con ozono ${ }^{29}$.

Atendiendo a la evolución de las variables estudiadas en el Índice de BODE ${ }^{26}$, el cual incluye el $\mathrm{FEV}_{1}$ (como medidor de la función pulmonar), la disnea (que representa la intensidad que acarrean los síntomas clínicos de la enfermedad) y la prueba de marcha en $6 \mathrm{~min}$, se puede decir que la ozonoterapia ejerció un efecto positivo en el mejoramiento de estas variables al término del tratamiento.

Teniendo en cuenta los resultados positivos alcanzados en este estudio, recomendamos la aplicación de la ozonoterapia como una variante terapéutica beneficiosa o como adyuvante al tratamiento convencional de pacientes con enfisema pulmonar.

\section{Referencias}

1. MacNee W, Carverly PMA. Chronic Obstructive Pulmonary Disease. 7: Management of COPD. Thorax 2003; 58: 261-5.

2. Braunwald E, Fauci AS, Kasper DL, Hauser SL, Longo DL, Jameson JL editores. Harrison. Principios de Medicina Interna. 15 Edición. Vol. 2. México: McGraw-Hill Interamericana; 2002. p. 1747-56.
3. Madison J, Irwin R. Chronic obstructive pulmonary disease. Lancet 1998; 352: 467-72.

4. Pauwels RA, Buist AS, Calverley PMA, Jenkins CR, Hurd SS. Global strategy for the diagnosis, management and prevention of chronic obstructive pulmonary disease. NHLBI/WHO global initiative for chronic obstructive lung disease (GOLD) workshop summary. Am J Respir Crit Care Med 2001; 163: 1256-76.

5. Shaker SB, Dirksen A, Ulrik CS, Hestad M, Stavngaard T, Laursen LC, et al. The effect of inhaled corticosteroids on the development of emphysema in smokers assessed by annual computed tomography. COPD 2009; 6: $104-$ 11.

6. Xu L, Cai BQ, Zhu YJ. Pathogenesis of cigarette smokeinduced chronic obstructive pulmonary disease and therapeutic effects of glucocorticoids and $\mathrm{N}$-acetylcysteine in rats. Chin Med J (Engl) 2004; 117: 1611-9.

7. Yamaguchi K, Matsubara H. Computed tomographic diagnosis of chronic obstructive pulmonary disease. Curr Opin Pulm Med 2000; 6: 92-8.

8. Piquette CA. Chronic bronchitis and emphysema. In Texbook of Respiratory medicine. $3^{\text {rd }}$ ed. Murray JF, Nadel JA, editors. Philadelphia, USA: Saunders; 2000. p. 1187 246.

9. Pearson M. BTS Guidelines for the management of chronic obstructive pulmonary disease. Thorax 1997; 52 (Suppl 5): S1-S5.

10. O’Byrne PM, Postma DS. The many faces of airway inflammation. Asthma and chronic obstructive pulmonary disease. Am J Resp Crit Care Med 1999; 159: S1-6.

11. Tetley TD. Inflammatory cells and chronic obstructive pulmonary disease. Curr Drug Targets Inflamm Allergy 2005; 4: 607-18.

12. Fiévez L, Kirschvink N, Zhang WH, Lagente V, Lekeux P, Bureau F, et al. Effects of betamethasone on inflammation and emphysema induced by cadmium nebulisation in rats. Eur J Pharmacol 2009; 606 (1-3): 210-4.

13. Menéndez S, González R, Ledea OE, Hernández F, León OS, Díaz M. Ozono. Aspectos básicos y aplicaciones clínicas. La Habana, Cuba: Editorial CENIC; 2008. p. 10-320.

14. Bocci V. A reasonable approach for the treatment of HIV infection in the early phase with ozonetherapy (autohemotherapy). How "inflammatory" cytokines may have a therapeutic role. Mediat Inflamm 1994; 3: 315-21.

15. Bette M, Mutters R, Nusing RM, Rodríguez ZZ, Menéndez S, Schulz S. Efficiency of Piperacillin/Tazobactam in lethal peritonitis is enhanced after preconditioning of rats with an oxidative stressor. Shock 2006; 25: 23-9.

16. León OS, Menéndez S, Merino N, Castillo R, Sam S, Pérez L, et al. Ozone oxidative preconditioning: a pro- 
tection against cellular damage by free radicals. Med Inflamm 1998; 7: 289-94.

17. Hernández F, Menéndez S, Wong R. Decrease of blood cholesterol and stimulation of antioxidative response in cardiopathy patients treated with endovenous ozone therapy. Free Radical Bio Med 1995; 19: 115-9.

18. Al-Dalain SM, Martínez G, Menéndez S, Re L, Giuliani A, Candelario-Jalil E, et al. Therapeutic efficacy of ozone medical treatments in patients with diabetic foot. Eur J Pharmacol 2005; 523: 151-61.

19. Zamora Z, Borrego A, López O, Delgado R, González R, Menéndez S, et al. Effects of ozone oxidative preconditioning on TNF-a release and antioxidant-prooxidant intracellular balance in mice during endotoxic shock. Mediat Inflamm 2005; 1: 16-22.

20. Calunga JL, Zamora ZB, Borrego A, del Río S, Barber E, Menéndez S, et al. Protective Effect of Ozone/Oxygen Mixture on Rats Submitted to Subtotal Nephrectomy: Role of Antioxidant System. Mediat Inflamm 2005; 4: 221-7.

21. Bocci V. Ozone. A new medical drug. AH Dordrecht, The Netherlands: Springer Publishers; 2005. p. 193-220.

22. Kirschvink N, Martin N, Fievez L, Smith N, Marlin D, Gustin P. Airway inflammation in cadmium-exposed rats is associated with pulmonary oxidative stress and emphysema. Free Radic Res 2006; 40: 241-50.

23. Ferrer M, Alonso J. Medición de la calidad de vida en los pacientes con EPOC. Arch Bronconeumol 2001; 37 (suppl): 20-6.

24. Celli B, Halpin D, Hepburn R, Byrne N, Golman M. Symptoms are an important outcome in chronic obs- tructive pulmonary disease clinical trials: result of a 3-month comparative study using the Breathlessness, Cough and Sputum Scale (BCSS). Respir Med 2003; 97 (suppl A): S35-43.

25. Jones PW, Quirk FH, Baveystock CM, Littlejons P. A selfcomplete measure of health status for chronic airflow limitation. The St. Georges Respiratory Questionaire. Am Rev Respir Dis 1992; 145: 1321-7.

26. Ramos P, Rodríguez JM. Diagnóstico y Monitorización. Biblioteca EPOC 2004; 2: 84-8.

27. Zayas K, González V, Calunga JL, Zamora Z. Efectos del ozono en un modelo de hipersensibilidad bronquial en cobayos. Revista Habanera de Ciencias Médicas (Cuba) 2006; 5(abril-junio): 2-3.

28. Rojas R, Martínez C, Turrent J, Menéndez S. Administración de ozono y reacción anafiláctica in vivo del cobayo. Revista CENIC Ciencias Biológicas (Cuba) 1999; 30: 119-20.

29. Hernández F, Calunga JL, Turrent J, Menéndez S, Montenegro A. Ozone therapy effects on blood biomarkers and lung function of asthma patients. Arch Med Res 2005; 36: 549-54.

30. Larini A, Bocci V. Effects of ozone on isolated peripheral blood mononuclear cells. Toxicol in Vitro 2005; 19: 55 61.

31. Stefanska AM, Walsh PT. Chronic obstructive pulmonary disease: evidence for an autoimmune component. Cell Mol Immunol 2009; 6: 81-6.

32. Guyton AC, Hall JE. Tratado de fisiología médica. 9a Edición. México: McGraw-Hill Interamericana; 1997. p. 323-40. 


\section{Referencias}

1. Martin W, Brynes S. Chilean Miners and Biomedical Research. A Modest Proposal. Am J Respir Crit Care Med 2010; 182: 1459-64.

2. Sobradillo P, Pozo F, Agustí A. Medicina P4: el futuro a la vuelta de la esquina. Arch Bronconeumol 2011; 47: 35-40.

Correspondencia a: Dr. Rafael Silva O.

Dos Norte 360, Talca. Fono: 71-412716 Fax: 71-209306

E-mail: rafaelsilvao@gmail.com 